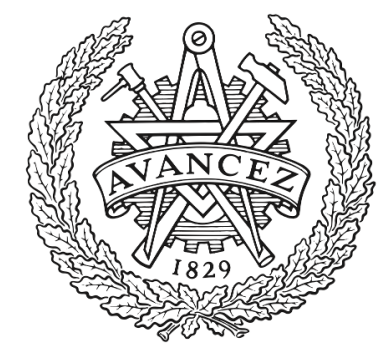

\title{
CHALMERS
}

UNIVERSITY OF TECHNOLOGY

\section{Elliptical-Core Highly Nonlinear Few-Mode Fiber Based OXC for WDM-MDM Networks}

Downloaded from: https://research.chalmers.se, 2023-04-26 02:41 UTC

Citation for the original published paper (version of record):

Gao, J., Nazemosadat Arsanjani, S., Yang, Y. et al (2021). Elliptical-Core Highly Nonlinear Few-Mode Fiber Based OXC for WDM-MDM Networks. IEEE Journal of Selected Topics in Quantum Electronics, 27(2). http://dx.doi.org/10.1109/JSTQE.2020.3012405

N.B. When citing this work, cite the original published paper.

(O2021 IEEE. Personal use of this material is permitted.

However, permission to reprint/republish this material for advertising or promotional purposes 


\title{
Elliptical-Core Highly Nonlinear Few-Mode Fiber Based OXC for WDM-MDM Networks
}

\author{
Jitao Gao, Elham Nazemosadat, Yi Yang, Songnian Fu, Ming Tang, Jochen Schröder, Magnus Karlsson, \\ and Peter A. Andrekson
}

\begin{abstract}
In order to realize an optical cross-connect (OXC) converting wavelengths and spatial modes into one-dimensional switching ports, we propose an active mode selective conversion without parasitic wavelength conversion, based on the intermodal four-wave mixing (FWM) arising in a few-mode fiber (FMF). First, we design a dispersion-engineered elliptical-core highly nonlinear FMF (e-HNL-FMF) with a graded refractive index (RI) profile, which can independently guide 3 linearly polarized (LP) spatial modes. Meanwhile, a high doping concentration of germanium in the core leads to relatively high intermodal nonlinear coefficients of $3.23(\mathrm{~W} \cdot \mathrm{km})^{-1}$ between $L P_{01}$ and $L P_{11 a}$ modes and $3.14(\mathrm{~W} \cdot \mathrm{km})^{-1}$ between $L P_{01}$ and $L P_{11 b}$ modes. Next, we propose an e-HNL-FMF based OXC scheme for wavelength division multiplexing-mode division multiplexing (WDM-MDM) networks. After optimizing both the e-HNL-FMF length and pump power, we can realize either active mode selective conversion over the designated wavelength-band or three-wavelength to three-mode superchannel conversion for 100 Gbaud 16-quadratic-amplitude modulation (16-QAM) signals over the C-band. Due to excellent characteristics of the e-HNL-FMF, both cost and configuration complexity of the OXC can be reduced, showing great potentials for all-optical signal processing in the future WDM-MDM networks.
\end{abstract}

Index Terms-Few-mode fiber, nonlinear fiber optics, four-wave mixing, optical cross-connect, optical signal processing.

\section{INTRODUCTION}

$\mathrm{A}$ s a well-known nonlinear phenomenon in optical fibers, four-wave mixing (FWM) has been extensively investigated. Owing to its advantages such as low-noise, ultrafast response and transparency to modulation formats, FWM has been widely applied in all-optical signal processing

This work was supported by National Key R\&D Program of China (2018YFB1801002) and National Natural Science Foundation of China (61875061). (Corresponding authors: Songnian Fu).

J. Gao, Y. Yang, S. Fu and M. Tang are with the Wuhan National Laboratory for Optoelectronics, and School of Optics and Electronic Information, Huazhong University of Science and Technology, Wuhan 430074, China (e-mail: gaojitao@hust.edu.cn; yangi@mail.hust.edu.cn; songnian@mail.hust.edu.cn; tangming@mail.hust.edu.cn).

E. Nazemosadat was with the Photonics Laboratory, Department of Microtechnology and Nanoscience, Chalmers University of Technology, Gothenburg 41296, Sweden, and currently with the ITEAM Research Institute, Universitat Politècnica de València, Valencia, 46022, Spain (e-mail: sbnazars@iteam.upv.es).

J. Schröder, M. Karlsson and P. A. Andrekson are with the Photonics Laboratory, Department of Microtechnology and Nanoscience, Chalmers University of Technology, Gothenburg 41296, Sweden (e-mail: jochen.schroeder@chalmers.se; peter.andrekson@chalmers.se). such as parametric amplification [1]-[2], wavelength conversion [3], wavelength exchange [4]-[6], all-optical regeneration [7]-[8], and optical switching [9]-[11]. Recently, rapid research progress in mode division multiplexing (MDM), an important category of space-division multiplexing (SDM), has been achieved to satisfy the ever-growing demand for communication capacity [12]-[13]. Hence, FWM arising in few-mode fibers (FMFs) has also gained worldwide research interests as a potential solution for all-optical signal processing applications, including few-mode parametric amplification [14]-[15], mode and wavelength conversion [16]-[23], and optical switching [24]. Those applications are either based on the intramodal FWM processes where all involved waves are within the same spatial mode [14]-[15], or intermodal FWM processes where the interacting waves are in different modes [16]-[23]. In comparison with the intramodal FWM processes requiring pumps to be close to the zero-dispersion wavelength for a wideband phase matching [25]-[26], intermodal FWM processes are more flexible in terms of implementation, as their phase-matching condition is mainly determined by the modal dispersion of each mode, which can be satisfied far away from the zero-dispersion wavelength [16], [27]. Bragg scattering (BS) and phase conjugation (PC) are mainly two intermodal FWM processes, where the energy transfers from pumps to both signal and idler for the PC process, while exchanging between signal and idler for the BS process [28]. The generated idler is both mode and wavelength converted with respect to the signal.

For future wavelength division multiplexing (WDM)-MDM networks, an optical cross-connect (OXC) which offers a selective conversion of both wavelength and mode channels is indispensable at all-optical network nodes. To date, the OXC for WDM-MDM networks is mainly based on complex interconnections of multiple mode/wavelength-division multiplexers/demultiplexers, which requires a large amount of optical components as well as complex control signals, leading to a relatively slow response time [29]. Alternatively, the function of active mode-selective conversion in OXC can be realized by the intermodal FWM processes, with a $\sim$ fs response time, flexible wavelength and mode selectivity, and convenient management. However, to the best of our knowledge, active mode-selective conversion occurs concurrently with wavelength conversion in all demonstrations of intermodal FWM to date [16]-[23], which makes them unsuitable for OXCs. Moreover, due to the wavelength-related phase-matching condition of intermodal FWM, an active 
mode-selective conversion can be realized with wavelength selectivity, indicating that it is possible to convert a WDM superchannel into a corresponding MDM superchannel. Given that the intermodal nonlinear coefficient is relatively low $(<0.9$ $\left.(\mathrm{W} \cdot \mathrm{km})^{-1}\right)$ in commercial FMFs [16], researchers have been motivated to develop highly nonlinear FMFs to enhance the conversion efficiency [21], [23]. Since an elliptical-core FMF can break the spatial degeneracy and allow two independent mode channels for the $\mathrm{LP}_{11}$ mode group, more degrees of freedom for optical signal processing based on intermodal FWM is anticipated [18].

In this submission, we propose an OXC scheme based on a specific intermodal FWM process, which can achieve mode-selective conversion without parasitic wavelength conversion, enabled by a new dispersion-tailored elliptical-core highly nonlinear FMF (e-HNL-FMF) design supporting 3 independent spatial modes, with intermodal nonlinear coefficients of $3.23(\mathrm{~W} \cdot \mathrm{km})^{-1}$ between $\mathrm{LP}_{01}$ and $\mathrm{LP}_{11 \text { a }}$ modes, and $3.14(\mathrm{~W} \cdot \mathrm{km})^{-1}$ between $\mathrm{LP}_{01}$ and $\mathrm{LP}_{11 \text { a }}$ modes. With the help of intermodal FWM arising in the e-HNL-FMF, the OXC can realize both active mode-selective conversion and three-wavelength to three-mode superchannel conversion for 100 Gbaud 16-QAM signals over the C-band. This paper is organized as follows: in Section II, the FWM process with the capability to perform active mode-selective conversion without the parasitic wavelength conversion is theoretically investigated, and the condition to achieve the complete mode-selective conversion is numerically identified. Based on the theoretical phase-matching condition, an e-HNL-FMF design is proposed in Section III. The dispersion properties and nonlinear coefficients of the e-HNL-FMF are numerically investigated for the active mode-selective conversion. In Section IV, an OXC scheme based on the e-HNL-FMF is proposed, and key parameters including fiber length and pump powers, are optimized for applications of active mode-selective conversion and three-wavelength to three-mode superchannel conversion. Finally, the corresponding performance is numerically evaluated with 100 Gbaud 16-QAM signals.

\section{THEORY OF ACTIVE MODE-SELECTIVE CONVERSION BASED ON INTERMODAL FWM}

The key technique of the proposed OXC for WDM-MDM networks is the active mode-selective conversion, which is based on intermodal FWM arising in the e-HNL-FMF. Generally, when two optical waves, pump $p$ at an angular frequency $\omega_{p}$ and signal wave $S$ at $\omega_{s}$, are coupled into the spatial mode $a$ of the e-HNL-FMF, while another optical wave, pump $q$ at $\omega_{q}$, is coupled into another spatial mode $b$, intermodal FWM processes occur in case the phase-matching conditions are satisfied. Idlers for BS and PC processes, $i_{\mathrm{BS}}$ and $i_{\mathrm{PC}}$, are generated in mode $b$ at

$$
\begin{aligned}
& \omega_{i, \mathrm{BS}}=\omega_{s}+\omega_{q}-\omega_{p} \\
& \omega_{i, \mathrm{PC}}=\omega_{p}+\omega_{q}-\omega_{s}
\end{aligned}
$$

as shown in Fig. 1(a). Meanwhile, another idler is generated at $\omega_{i, \mathrm{MI}}=2 \omega_{p}-\omega_{s}$ by the intramodal FWM process, known as modulational instability (MI). Through BS and PC, $i_{\mathrm{BS}}$ and $i_{\mathrm{PC}}$ are mode-converted with respect to $S$, and this mode conversion is accompanied by a parasitic wavelength conversion, as observed in Eqs. (1) and (2). Moreover, the phase mismatches of $\mathrm{BS}$ and $\mathrm{PC}$ processes are $\Delta \beta_{\mathrm{BS}}=\beta^{a}\left(\omega_{s}\right)+\beta^{b}\left(\omega_{q}\right)-\beta^{a}\left(\omega_{p}\right)-\beta^{b}\left(\omega_{i, \mathrm{BS}}\right) \quad$ and $\Delta \beta_{\mathrm{PC}}=\beta^{a}\left(\omega_{s}\right)+\beta^{b}\left(\omega_{i, \mathrm{PC}}\right)-\beta^{a}\left(\omega_{p}\right)-\beta^{b}\left(\omega_{q}\right)$, respectively, where $\beta^{m}(\omega)$ represents the propagation constant $\beta$ of mode $m$ at angular frequency $\omega$. If we expand the items related to $\beta^{a}$ at $\left(\omega_{p}+\omega_{s}\right) / 2$ and the items related to $\beta^{b}$ at $\left(\omega_{q}+\omega_{i}\right) / 2$ in a Taylor series, respectively, ignoring the contribution of higher order dispersion, the phase mismatch for both BS and PC processes can be obtained as [16]

$$
\Delta \beta_{\mathrm{BS} / \mathrm{PC}} \approx\left(\omega_{s}-\omega_{p}\right)\left[\beta_{1}^{a}\left(\frac{\omega_{s}+\omega_{p}}{2}\right)-\beta_{1}^{b}\left(\frac{\omega_{i}+\omega_{q}}{2}\right)\right]
$$

where $\beta_{1}^{m}(\omega)$ represents the inverse group velocity of mode $m$ at angular frequency $\omega, i$ represents $i_{\mathrm{BS}}$ or $i_{\mathrm{PC}}$, depending on the FWM process to be investigated. Both BS and PC processes occur, when the phase-mismatch is close to zero. Thus, the FWM effects on $s$ and $i$ are wavelength-sensitive, with their individual wavelength ranges centered at $\omega_{s}$ and $\omega_{i}$, respectively. However, for the OXC application, two center wavelengths should be the same, indicating of that the aforementioned parasitic wavelength conversion needs to be suppressed during the active mode-selective conversion. Therefore, we assume $\omega_{i}=\omega_{s}$, which means $\omega_{p}=\omega_{q}$ for the BS process referred as the wavelength-degenerate BS (WD-BS) process, and $\omega_{p}+\omega_{q}=2 \omega_{s}$ for the PC process, respectively.

For the purpose of active mode-selective conversion, the idler should copy the signal properties, which means it should only participate in the photon exchange process with the signal, and

(a)

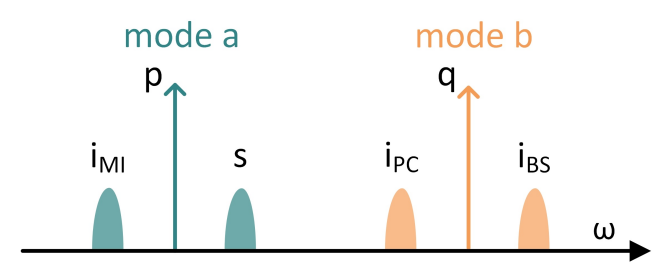

(b)

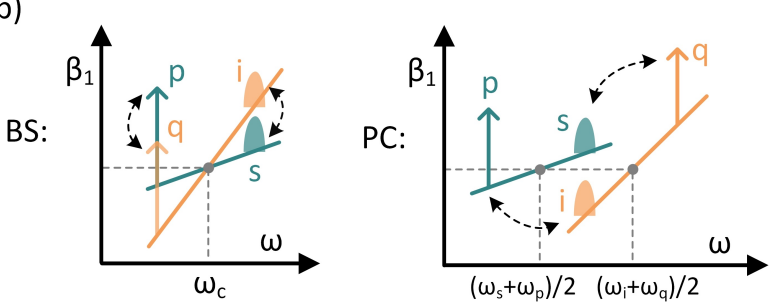

Fig. 1. (a) Schematic of the intermodal and intramodal FWM processes. (b) Schematic of mode conversion based on BS and PC processes, different colors represent different modes. Double-ended arrows represent how the photons exchange. 
not the pumps [4]. Out of two intermodal FWM processes, only the WD-BS process can be used to satisfy this condition, because in the PC process, both the signal and idler exchange photons with the pumps and get amplified or depleted simultaneously, as shown in Fig. 1(b). As a result, the phase-matching condition of the WD-BS process is

$$
\Delta \beta_{\mathrm{WD}-\mathrm{BS}}=\left(\omega_{s}-\omega_{p}\right)\left[\beta_{1}^{a}\left(\omega_{c}\right)-\beta_{1}^{b}\left(\omega_{c}\right)\right]=0
$$

where $\omega_{c}=\left(\omega_{p}+\omega_{s}\right) / 2=\left(\omega_{q}+\omega_{i}\right) / 2$ represents the center angular frequency of two interacting waves in each mode. This means that $\beta_{1}^{a}\left(\omega_{c}\right)=\beta_{1}^{b}\left(\omega_{c}\right)$. In other words, $\beta_{1}$ of two interacting modes must have an intersection at $\omega_{c}$. Accordingly, for practical implementations, if $\beta_{1}$ of two modes within a given FMF are measured to be intersecting at $\omega_{0}$, in order to mode-convert an input signal at $\omega_{s}$, one should launch a pair of pump waves into both the initial and target modes at $\omega_{p}=2 \omega_{0}-\omega_{s}$. That is to say, we can intentionally set $\omega_{c}=\omega_{0}$ to satisfy the phase-matching condition of the mode conversion. Thus, for any given signal, by appropriately adjusting the pump wavelengths, such that $\omega_{c}=\omega_{0}$, wavelength-selective mode conversion can be achieved. Meanwhile, active mode-selective conversion can also be achieved when $\beta_{1}$ curves of all modes arising in the FMF intersect at wavelengths which are close to each other. Furthermore, the mode conversion bandwidth (BW) for a given wavelength channel becomes larger, when the pumps and signal are close to $\omega_{c}$, because the phase mismatch $\Delta \beta_{\text {WD-BS }}$ is proportional to the small drift of signal wavelength. To achieve wide mode conversion BW over a target operation wavelength range (e.g. C-band), the intersecting wavelength should be designed close to the center of the operation wavelength range.

The optical power transferred from signal to idler depends on the nonlinear phase-shift $\phi_{N L}$ induced by the WD-BS process. Assuming the continuous-wave (CW) operation under the small-signal regime and ignoring the MI effect, which can be designed to be phase-mismatched in the FMF, the coupled nonlinear equations for the WD-BS process are [6]

$$
\begin{gathered}
\frac{\partial A_{p}^{a}}{\partial z}=i\left(\gamma_{a a}\left|A_{p}^{a}\right|^{2}+2 \gamma_{a b}\left|A_{p}^{b}\right|^{2}\right) A_{p}^{a} \\
\frac{\partial A_{p}^{b}}{\partial z}=i\left(\gamma_{b b}\left|A_{p}^{b}\right|^{2}+2 \gamma_{a b}\left|A_{p}^{a}\right|^{2}\right) A_{p}^{b} \\
\frac{\partial A_{s}^{a}}{\partial z}=i\left(2 \gamma_{a a}\left|A_{p}^{a}\right|^{2}+2 \gamma_{a b}\left|A_{p}^{b}\right|^{2}\right) A_{s}^{a}+2 i \gamma_{a b} A_{p}^{a} A_{p}^{b *} A_{i}^{b} e^{-i \Delta \beta_{\mathrm{WD}-\mathrm{BS}} z} \\
\frac{\partial A_{i}^{b}}{\partial z}=i\left(2 \gamma_{a b}\left|A_{p}^{a}\right|^{2}+2 \gamma_{b b}\left|A_{p}^{b}\right|^{2}\right) A_{i}^{b}+2 i \gamma_{a b} A_{p}^{a *} A_{p}^{b} A_{s}^{a} e^{i \Delta \beta_{\mathrm{WD}-\mathrm{BS}}}
\end{gathered}
$$

where $A_{x}^{m}$ is the complex field amplitude of mode $m$ at the wavelength of wave $x$, and $\gamma_{m n}$ is the nonlinear coefficient between modes $m$ and $n$ [26]. Here, we use $A_{p}^{b}$ instead of $A_{q}^{b}$ for the ease of expression since $p$ and $q$ are wavelength-degenerate. By solving Eqs. (3)-(7), assuming a phase-matched process ( $\Delta \beta_{\text {wD-Bs }}+\gamma_{a a} P_{p}^{a}-\gamma_{b b} P_{p}^{b}=0$, considering the phase shift caused by the cross-phase modulation), the powers of the signal and idler at arbitrary longitudinal location $z$ are [4], [5]

$$
\begin{aligned}
& P_{i}^{b}(z)=P_{s}^{a}(0) \sin ^{2}\left(\gamma_{a b} P_{p} z\right) \\
& P_{s}^{a}(z)=P_{s}^{a}(0) \cos ^{2}\left(\gamma_{a b} P_{p} z\right)
\end{aligned}
$$

respectively, where $P_{p}=P_{p}^{a}+P_{p}^{b}$ represents the total pump power. Note that we normally consider the phase-matched WD-BS process and equal pump powers ( $\Delta \beta_{\text {WD-BS }}=0$ and $P_{p}^{a}=P_{p}^{b}$ ), which induces a small blue shift to the perfect phase-matched signal wavelength and can be easily mitigated by adjusting the pump wavelength. Eqs. (9) and (10) indicate that the signal and idler powers vary periodically with $z$, while the total power of signal and idler remains constant. Thus, no optical power is transferred from the pumps to the signal or idler. Furthermore, complete power conversion from signal to idler can be achieved when the nonlinear phase shift is $\phi_{N L}=\gamma_{a b} P_{p} z=(2 n+1) \pi / 2$, where $n$ is an integer. Consequently, for different combinations of $P_{p}$ and FMF length $L$ satisfying $\phi_{N L}=(2 n+1) \pi / 2$, active mode conversion can be always achieved at the phase-matched signal wavelength. However, the conversion $\mathrm{BW}$ and the mainlobe-to-sidelobe power ratio (MSPR), which is defined by the power ratio between the mainlobe peak and the largest sidelobe peak for the parametric conversion spectrum, differ from case to case. In order to satisfy the OXC demand on the conversion BW and MSPR optimization of $L$ and $P_{p}$ is necessary.

\section{ELLIPTICAL-CORE HIGHLY NONLINEAR FMF DESIGN}

We propose a dispersion-engineered e-HNL-FMF design with a graded refractive index (RI) elliptical core together with an ultra-low RI trench, as shown in Fig. 2(a). The RI profiles of the major and minor axes of the designed e-HNL-FMF are shown in Fig. 3. With the help of the elliptical core, which breaks the spatial degeneracy of the $\mathrm{LP}_{11}$ mode group, the e-HNL-FMF supports 3 independent LP modes, namely $\mathrm{LP}_{01}$, (a)

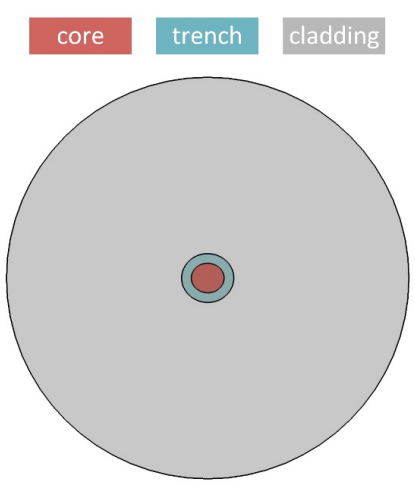

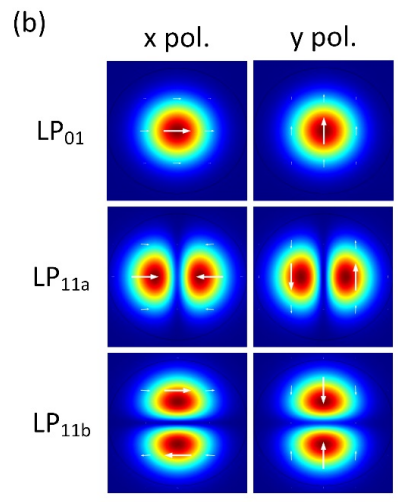

Fig. 2. (a) Transverse section of the e-HNL-FMF. (b) Power distributions and polarization distributions of three guided LP modes. 
$\mathrm{LP}_{11 \mathrm{a}}$ and $\mathrm{LP} \mathrm{P}_{11 \mathrm{~b}}$, as shown in Fig. 2(b). The relative effective RI difference $\Delta \mathrm{n}_{\text {eff }}$ between any two modes is more than $1.2 \times 10^{-3}$, as shown in the inset of Fig. 3. Fiber nonlinearity is important for the e-HNL-FMF to effectively perform the intermodal FWM, which can be evaluated by the nonlinear coefficient of $\gamma=\omega n_{2} /\left(c A_{\text {eff }}\right)$, where $n_{2}$ represents the equivalent nonlinear RI over the core area according to the expression provided in [30], $C$ represents the speed of light in vacuum, and $A_{\text {eff }}$ represents the effective mode area. In order to enhance the nonlinearity, the core area is highly doped with germanium (32 mol.\% at the center of elliptical core, with an RI profile exponent $\alpha$ of 1.86), and the semi-major and semi-minor axes of elliptical core are restricted to $5.1 \mu \mathrm{m}$ and $4.6 \mu \mathrm{m}$, respectively, in order to reduce the effective mode field area. The effective areas of $\mathrm{LP}_{01}, \mathrm{LP}_{11 \mathrm{a}}$ and $\mathrm{LP}_{11 \mathrm{~b}}$ modes are 15.9, 21.9 and $22.0 \mu \mathrm{m}^{2}$, respectively. Meanwhile, the intramodal nonlinear coefficients are $6.44(\mathrm{~W} \cdot \mathrm{km})^{-1}, 4.66(\mathrm{~W} \cdot \mathrm{km})^{-1}$ and $4.64(\mathrm{~W} \cdot \mathrm{km})^{-1}$ for $\mathrm{LP}_{01}, \mathrm{LP}_{11 \mathrm{a}}$, and $\mathrm{LP}_{11 \mathrm{~b}}$ modes, respectively. By calculating the overlap integral of the spatial distribution between any two LP modes, the intermodal $A_{\text {eff }}$ values are found to be $31.7 \mu \mathrm{m}^{2}$ between $\mathrm{LP}_{01}$ and $\mathrm{LP}_{11 \mathrm{a}}, 32.5 \mu \mathrm{m}^{2}$ between $L P_{01}$ and $L P_{11 b}$, and $63.2 \mu \mathrm{m}^{2}$ between $L P_{11 a}$ and $L P_{11 b}$.

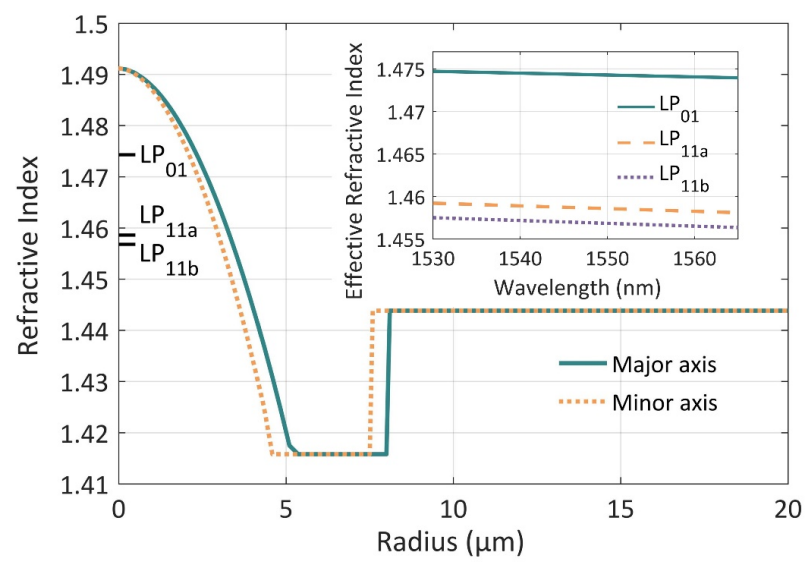

Fig. 3. RI profile of the proposed e-HNL-FMF. The black marks represent the effective RIs of all guided modes at $1550 \mathrm{~nm}$. Inset: Effective RI of guided modes over the C-band.

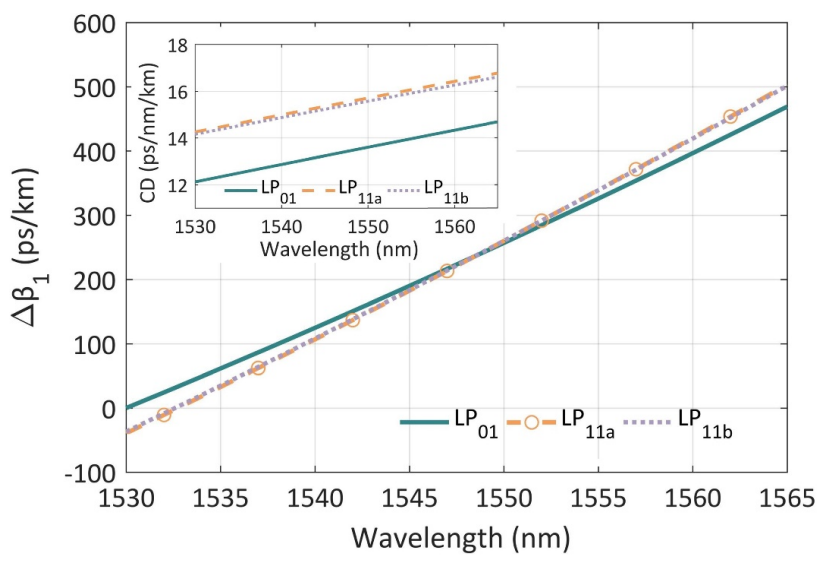

Fig. 4. Relative inverse group velocity and CD (inset) of the guided modes versus wavelength.
Accordingly, the intermodal nonlinear coefficients $\gamma_{01,11 a}$, $\gamma_{01,11 \mathrm{~b}}$ and $\gamma_{11 \mathrm{a}, 11 \mathrm{~b}}$ (the subscript of $\gamma$ represents interacting LP modes) are $3.23(\mathrm{~W} \cdot \mathrm{km})^{-1}, 3.14(\mathrm{~W} \cdot \mathrm{km})^{-1}$, and $1.62(\mathrm{~W} \cdot \mathrm{km})^{-1}$, respectively.

According to the mode conversion theory of the WD-BS process, $\beta_{1}$ curves of all spatial modes must have intersections at the same wavelength, which is ideally near the center of the C-band. However, the elliptical core breaks the spatial degeneracy of the $\mathrm{LP}_{11}$ modes, leading to walk-off between $\mathrm{LP}_{11 \mathrm{a}}$ and $\mathrm{LP}_{11 \mathrm{~b}}$ modes, which causes a shift between their $\beta_{1}$ curves. By optimizing $\alpha$, the $\beta_{1}$ difference between $\mathrm{LP}_{01}$ and $\mathrm{LP}_{11}$ mode groups can be reduced. Moreover, by optimizing the core radius and ellipticity, along with the width and the fluorine doping concentration of the trench, $\beta_{1}$ curves of $\mathrm{LP}_{11 \mathrm{a}}$ and $\mathrm{LP}_{11 \mathrm{~b}}$ can be very close to each other without losing the mode independence. The optimal width of the trench is $3.0 \mu \mathrm{m}$, while its RI is 1.416. As shown in Fig. 4, $\beta_{1}$ curves of all spatial modes intersect at $1548.45 \mathrm{~nm}$, almost at the center of the C-band, which not only enables active mode-selective conversion but also provides a large mode conversion BW over the C-band. However, as shown in [23], $\beta_{1}$ curves of all spatial modes are very sensitive to the geometric parameters of core. Thus, assuming a fabrication accuracy of core radius of 0.01 $\mu \mathrm{m}$, three spatial modes do not intersect exactly at the same wavelength. $\beta_{1}$ curves of $\mathrm{LP}_{01}$ and $\mathrm{LP}_{11 \mathrm{a}}$ modes intersect at $\omega_{01,11 \mathrm{a}}=1548.55 \mathrm{~nm}$, while for $\mathrm{LP}_{01}$ and $\mathrm{LP}_{11 \mathrm{~b}}$, the intersection is $\omega_{01,11 \mathrm{~b}}=1548.37 \mathrm{~nm}$. In addition, $\beta_{1}$ curves of $\mathrm{LP}_{11 \mathrm{a}}$ and $\mathrm{LP}_{11 \mathrm{~b}}$ modes are very close to each other, which enables a large conversion BW of BS process between the modes [27]. For any given pump and signal wavelength, apart from the WD-BS process, both PC and MI processes occur as well, which generate another idler $j$ in both involved modes at $\omega_{j}=2 \omega_{p}-\omega_{s}$. Therefore, the cascaded FWM processes that are seeded by $j$ also need to be considered, namely WD-BSc, PCc and MIc, as shown in Table I. The phase-matching condition for the intramodal FWM process in each mode is $\Delta \beta_{\mathrm{MI}} \approx\left(\omega_{s}-\omega_{p}\right)^{2} \beta_{2}\left(\omega_{p}\right)$ [26], which is determined by the chromatic dispersion (CD) of each mode. For the given pump wavelengths of $1540.2 \mathrm{~nm}$ and $1539.8 \mathrm{~nm}$ and for mode conversion from $\mathrm{LP}_{01}$ to $\mathrm{LP}_{11 \mathrm{a}}$ and $\mathrm{LP}_{11 \mathrm{~b}}$ modes, respectively,

TABLE I

FWM PROCESSES CONSIDERED IN MODE-SELECTIVE CONVERSION

\begin{tabular}{|c|c|c|}
\hline $\begin{array}{c}\text { FWM } \\
\text { No. }\end{array}$ & Type of FWM & FWM process $^{a}$ \\
\hline WD-BS & Intermodal, BS & $p^{11 \mathrm{a} / \mathrm{b}}+s^{01} \rightarrow p^{01}+i^{11 \mathrm{a} / \mathrm{b}}$ \\
\hline $\mathrm{PC}$ & Intermodal, PC & $p^{01}+p^{11 \mathrm{a} / \mathrm{b}} \rightarrow s^{01}+j^{11 \mathrm{a} / \mathrm{b}}$ \\
\hline MI & Intramodal, MI & $2 p^{01} \rightarrow s^{01}+j^{01}$ \\
\hline WD-BSc & Intermodal, BS, cascaded & $p^{11 \mathrm{a} / \mathrm{b}}+j^{01} \rightarrow p^{01}+j^{11 \mathrm{a} / \mathrm{b}}$ \\
\hline PCc & Intermodal, PC, cascaded & $p^{01}+p^{11 \mathrm{a} / \mathrm{b}} \rightarrow j^{01}+i^{11 \mathrm{a} / \mathrm{b}}$ \\
\hline MIc & Intramodal, MI, cascaded & $2 p^{11 \mathrm{a} / \mathrm{b}} \rightarrow i^{11 \mathrm{a} / \mathrm{b}}+j^{11 \mathrm{a} / \mathrm{b}}$ \\
\hline
\end{tabular}

${ }^{\text {a}}$ For each process, the superscript of each wave is the LP mode which the wave is in. 


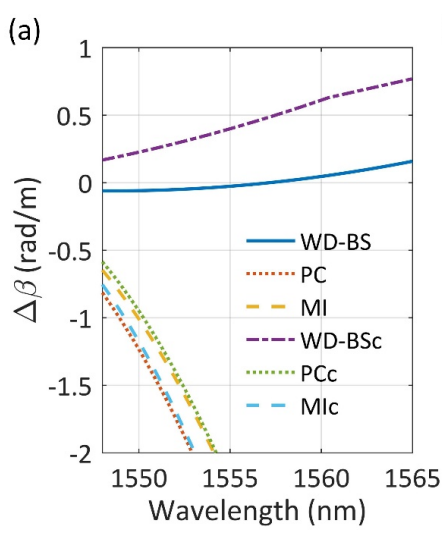

(b)

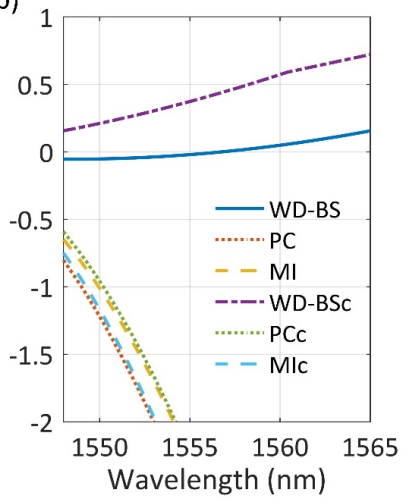

Fig. 5. Phase mismatch of the 6 FWM processes in active mode-selective conversion between (a) $\mathrm{LP}_{01}$ and $\mathrm{LP}_{11 \mathrm{a}}$ modes when pump wavelength is 1540.2 $\mathrm{nm}$, and (b) $\mathrm{LP}_{01}$ and $\mathrm{LP}_{11 \mathrm{~b}}$ modes when pump wavelength is $1539.8 \mathrm{~nm}$.

the phase mismatches of 6 FWM processes with respect to the signal wavelength are shown in Fig. 5. We can clearly observe that only when the signal wavelength is $1557 \mathrm{~nm}$, is the WD-BS process phase-matched, while the PC, MI, PCc and MIc processes have large phase mismatches. The occurrence of intramodal FWM processes is challenging, because CD of each mode is far from zero over the C-band, as shown in the inset of Fig. 4. Therefore, those processes can be ignored in the theoretical investigations of Section II, when the signal and pump wavelengths are not very close to each other. As for the WD-BSc process between $p$ and $j$, although the phase-mismatch is smaller in comparison with the PC and MI processes, it is considerably larger than the WD-BS process. In order to prevent the occurrence of the non-essential FWM processes, the pump wavelength can be intentionally shifted several nanometers away from the signal, thanks to the large conversion BW of the WD-BS process, as shown in later Fig. 9. Moreover, since the power of $j$ in both modes generated by MI and PC processes is very low and no amplification of $j$ will be introduced by the WD-BSc process itself, the WD-BSc process can also be ignored.

Due to the specific dispersion properties of e-HNL-FMF, it can be used for OXC application in WDM-MDM networks. Signal in a given mode can be converted to an arbitrary mode without parasitic wavelength conversion, as shown in Fig. 6(a), when a pair of pumps at the corresponding wavelength are launched into both initial and target modes. Complete (a)

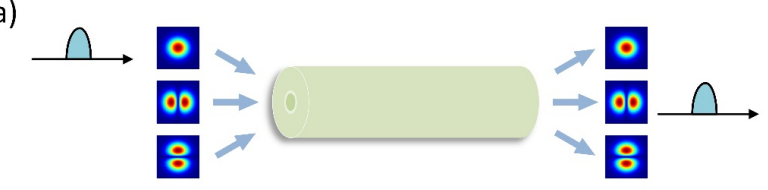

(b)

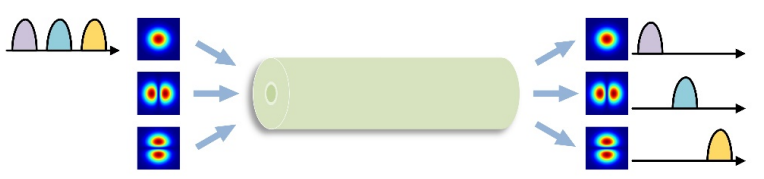

Fig. 6. Schematic of applications based on e-HNL-FMF: (a) active mode-selective conversion, and (b) three-wavelength to three-mode superchannel conversion.

mode-selective conversion can be achieved when both the length of e-HNL-FMF and the pump power are optimized. It is noteworthy that the active mode-selective conversion is wavelength tunable, because for any given signal wavelength over the C-band, the pump wavelengths can be adjusted accordingly to satisfy the phase-matching condition. Furthermore, with the help of the wavelength-selectivity, the e-HNL-FMF supports the conversion from a three-wavelength superchannel signal to a three-mode superchannel signal, as shown in Fig. 6(b). This can be achieved by sending multiple pairs of pumps into the initial and target modes corresponding to each signal. Other applications such as wavelength conversion and one-to-three mode multicasting can also be realized through the intermodal FWM processes arising in the e-HNL-FMF. In summary, the designed e-HNL-FMF enables flexible OXC application by simply tuning the powers and wavelengths of the pumps. The e-HNL-FMF based OXC can potentially have large mode conversion BW with ultrafast response and is transparent to modulation formats. Moreover, the complexity of OXC can be reduced in comparison with current WDM-MDM OXC structures.

\section{SYSTEM SETUP AND SIMULATION RESULTS}

Based on the optical properties of the e-HNL-FMF, we numerically investigate the performance of the e-HNL-FMF based OXC for active mode-selective conversion and three-wavelength to three-mode superchannel signal conversion applications. The OXC setup is schematically shown in Fig. 7. A three-wavelength WDM superchannel with equal frequency spacing, referred to as Signal1, Signal2 and

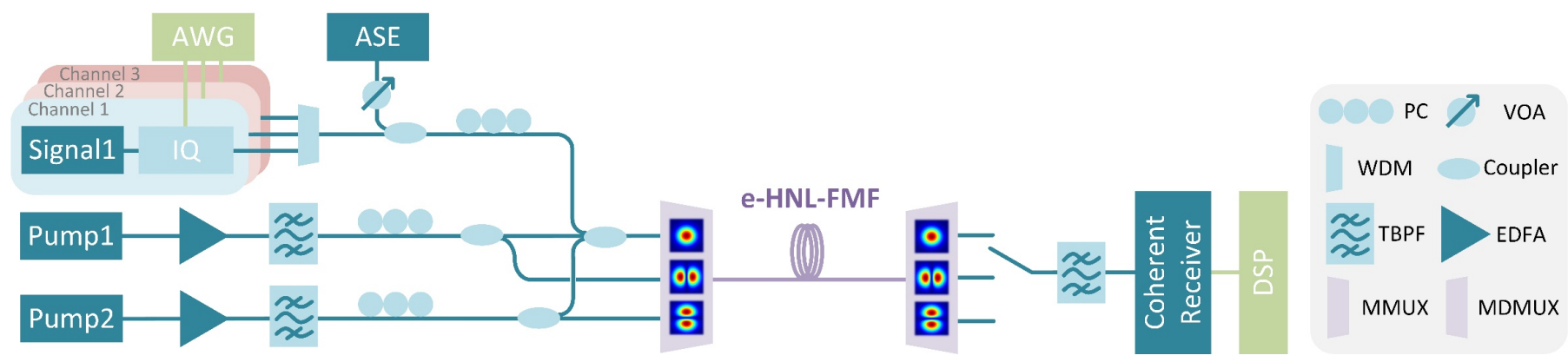

Fig. 7. System setup of the OXC based on the designed e-HNL-FMF. Abbreviations not included in main text: PC: Polarization controller; VOA: Variable optical attenuator; WDM: Wavelength-division multiplexer; DSP: Digital signal processing. 
Signal3, is generated by three tunable CW lasers and coupled together by a wavelength-division multiplexer. Each signal can be modulated with 16-QAM at a symbol rate of 25 or 100 Gbaud, with the help of an IQ modulator driven by an arbitrary waveform generator (AWG). Amplified spontaneous emission (ASE) noise is introduced to the WDM signals before launching them into the $\mathrm{LP}_{01}$ mode of the e-HNL-FMF. Two pumps (Pump1 and Pump2) are generated by tunable CW lasers, then separately amplified by an erbium-doped fiber amplifier (EDFA). The out-of-band ASE noise introduced by EDFAs is filtered by tunable band-pass filters (TBPFs). Then, each pump propagates through a coupler and is launched into two spatial modes of the e-HNL-FMF, which are $\mathrm{LP}_{01}$ and $\mathrm{LP}_{11 \text { a }}$ modes for Pump1, and $\mathrm{LP}_{01}$ and $\mathrm{LP}_{11 \mathrm{~b}}$ modes for Pump2, respectively. The pumps to be launched into $\mathrm{LP}_{01}$ mode are coupled together with the signals. Before multiplexing all waves into the e-HNL-FMF, they are aligned in polarization. The mode-division multiplexer (MMUX) and demultiplexer (MDMUX) are mode-selective and assumed to be ideal, which means there is no crosstalk among three mode channels, and the mode-dependent loss is zero. During the propagation over the e-HNL-FMF, a scalar 3-mode model based on coupled-wave equations is used to investigate the wave interaction, which are numerically solved by a symmetrized split step Fourier method. After MDMUX, signals or idlers generated by FWMs in each mode are received and analyzed. As for the e-HNL-FMF, we need to optimize the parameters including the length of e-HNL-FMF and the powers of two pumps, which may be different for different applications.

\section{A. Active mode-selective conversion}

For active mode-selective conversion, we choose a single wavelength signal and use Pump1 or Pump2 for the conversion from $L P_{01}$ mode to $L P_{11 a}$ and $L P_{11 b}$ modes, respectively. For a given signal angular frequency $\omega_{s}$ in $\mathrm{LP}_{01}$ mode, we set Pump1 at $2 \omega_{01,11 \mathrm{a}}-\omega_{s}$ or Pump2 at $2 \omega_{01,11 \mathrm{~b}}-\omega_{s}$, depending on the target mode. As mentioned in Section II, complete

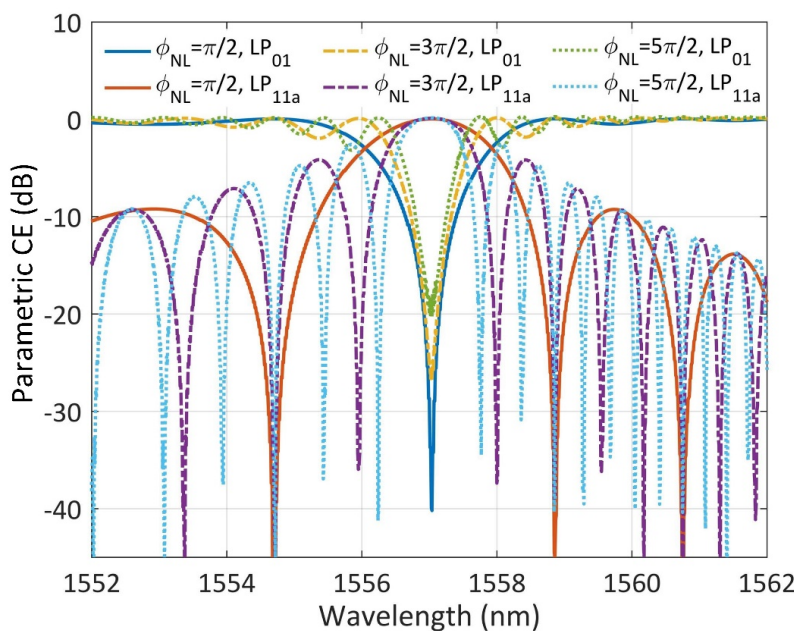

Fig. 8. Parametric CE spectra for mode conversion from $\mathrm{LP}_{01}$ mode to $\mathrm{LP}_{11 \mathrm{a}}$ mode when pump wavelength is $1540.2 \mathrm{~nm}$, under same pump power but different fiber length to obtain nonlinear phase shifts of $\pi / 2,3 \pi / 2$ and $5 \pi / 2$, respectively. mode-selective conversion can be achieved when the nonlinear phase shift $\phi_{N L}=\gamma_{01,11 \mathrm{a} / \mathrm{b}} P_{p} L$ is equal to $(2 n+1) \pi / 2$, where $L$ represents the fiber length. To optimize $P_{p}$ and $L$, we investigate the parametric conversion efficiency (CE), which is determined by the power ratio between the output signal (idler) and the input signal, as well as the 3-dB BW of active mode-selective conversion over the C-band, by simulating the propagation of the $\mathrm{CW}$ signal without the data loading. Please note that all 6 FWM processes in Table I are taken into account. Since the active mode conversion from $\mathrm{LP}_{01}$ mode to $\mathrm{LP}_{11 \mathrm{a}}$ and $\mathrm{LP}_{11 \mathrm{~b}}$ modes are similar, we only present the optimization of $P_{p}$ and $L$ based on the conversion to $\mathrm{LP}_{11 \mathrm{a}}$. Accordingly, the optimal $P_{p}$ for the conversion to $\mathrm{LP}_{11 \mathrm{~b}}$ mode can be calculated, based on $\gamma_{01,11 \mathrm{a}}$ and $\gamma_{01,11 \mathrm{~b}}$. With the same $P_{p}$, if we change $L$ to set $\phi_{N L}$ equal to $\pi / 2,3 \pi / 2$ and $5 \pi / 2$, respectively, the corresponding parametric CE spectra of the signal and idler are shown in Fig. 8. If we treat the parametric CE spectrum of idler in $\mathrm{LP}_{11}$ a mode as an optical filter response, as $\phi_{N L}$ increases, the mainlobe (passband) of the idler becomes narrower. As a result, the mode conversion BW becomes limited. The maximum mode conversion efficiency (MCE), which is determined by power ratio between the target mode and the initial mode, also degrades with larger $\phi_{N L}$. Nevertheless, the power of the sidelobes increases, leading to the reduction of MSPR. Consequently, the negligible depletion of signal power at sidelobe wavelengths occurs. Therefore, in order to enlarge the mode conversion $\mathrm{BW}$ and obtain the optimal performance of the stopband, we choose $\pi / 2$ as the target $\phi_{N L}$ value. Then, for a given $P_{p}$, there exists a certain value of $L$ to satisfy $\phi_{N L}=\pi / 2$. Thus, we can investigate the mode conversion BW with respect to the target signal wavelength and $P_{p}$, as shown in Fig. 9. Please note that the pump wavelength is also optimized for each case in Fig. 9 for

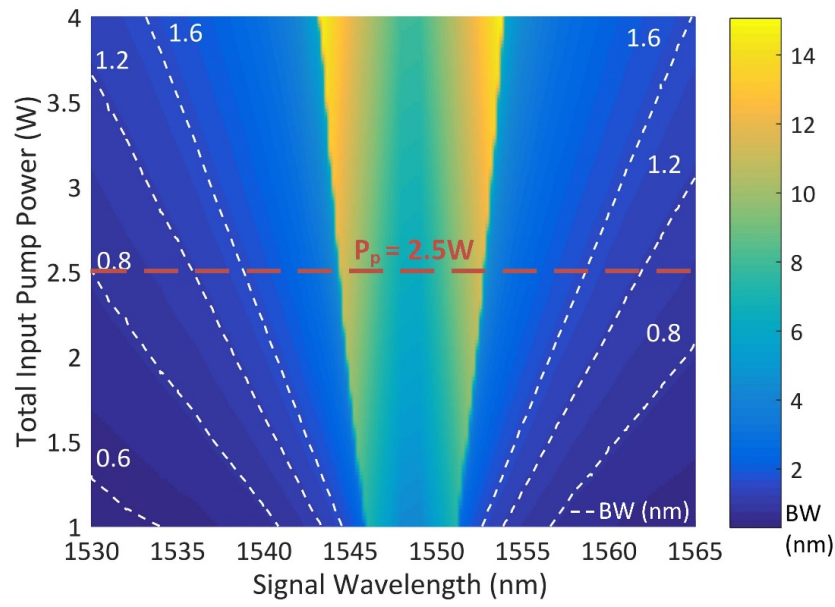

Fig. 9. Mode conversion $\mathrm{BW}$ between $\mathrm{LP}_{01}$ and $\mathrm{LP}_{11 \mathrm{a}}$ modes, with respect to the target signal wavelengths and total pump power. For every target signal wavelength, the pump wavelength and fiber length are adjusted accordingly to realize the complete mode conversion. The BW smaller than $2 \mathrm{~nm}$ is illustrated with the white dashed contour lines for the ease of observation. The red dashed line represents the optimal pump power. 
fulfilling the phase-matching condition. For every target signal wavelength, the $3-\mathrm{dB}$ mode conversion $\mathrm{BW}$ increases with the growing $P_{p}$. Since the $\beta_{1}$ curves of $\mathrm{LP}_{01}$ and $\mathrm{LP}_{11 \mathrm{a}}$ are not parallel, the BWs are relatively limited in comparison with other FMFs for the wavelength conversion [19], [27], and indicate of wavelength dependence. There is a region where $\mathrm{BW}$ is larger than $4 \mathrm{~nm}$ when signal wavelength is relatively close to $1548.45 \mathrm{~nm}$, while the $\mathrm{BW}$ decreases when signal wavelength is out of the region. When $P_{p}$ is $2.5 \mathrm{~W}(1.25 \mathrm{~W}$ in each mode), the mode conversion $\mathrm{BW}$ over the $\mathrm{C}$-band is larger than $0.8 \mathrm{~nm}$, which means the e-HNL-FMF based OXC has the capability to convert signals up to $100 \mathrm{Gbaud}$ symbol rate. The corresponding $L$ is $195 \mathrm{~m}$, and for the conversion from $L P_{01}$ to $\mathrm{LP}_{11 \mathrm{~b}}$ mode the corresponding $P_{p}$ is $2.57 \mathrm{~W}$. The relatively high pump power may cause stimulated Brillouin scattering (SBS), which can be suppressed by employing the pump frequency dithering technique during the experimental implementation [6]. Please note that for a signal with specific wavelength and baud rate, there always occurs an optimal $P_{p}$

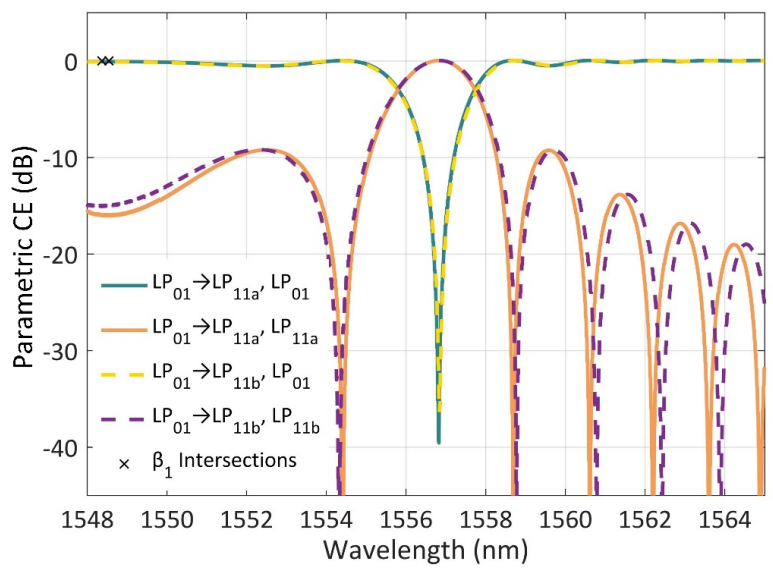

Fig. 10. Parametric CE spectrum of each involved mode referred to input signal power, when the target signal wavelength is $1557 \mathrm{~nm}$. The solid lines represent mode conversion from $\mathrm{LP}_{01}$ to $\mathrm{LP}_{11}$, while the dashed lines represent mode conversion from $L P_{01}$ to $\mathrm{LP}_{11 \mathrm{~b}}$.

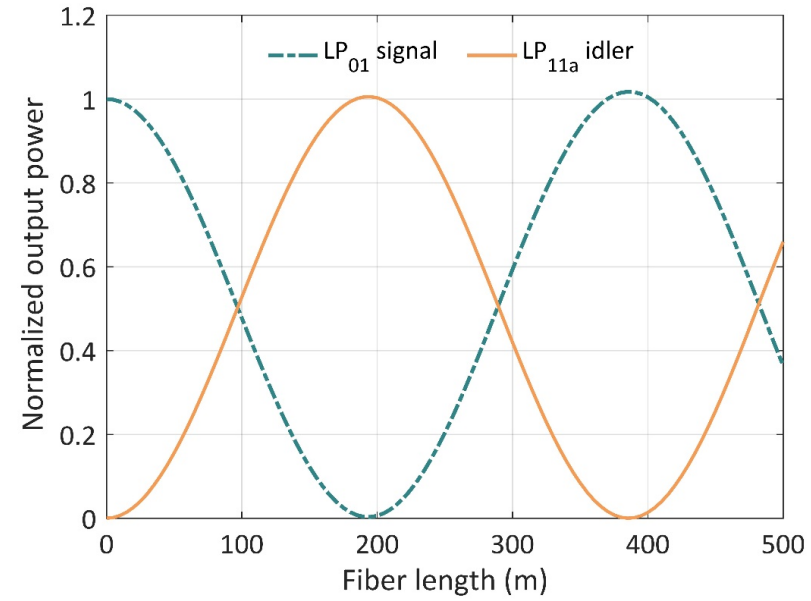

Fig. 11. Power evolution of the signal and idler with respect to the longitudinal propagation distance. with a corresponding $L$. If the signal has relatively low symbol rate or its wavelength is relatively close to $1548.45 \mathrm{~nm}$, it is possible to use a lower $P_{p}$ with a longer $L$. With the optimal $L$ and $P_{p}$, we are able to calculate the parametric CE spectrum of mode conversion at a given target signal wavelength (e.g. $1557 \mathrm{~nm}$, which means the wavelengths of Pump1 and Pump2 are $1540.2 \mathrm{~nm}$ and $1539.8 \mathrm{~nm}$, respectively), as shown in Fig. 10. Signal in $\mathrm{LP}_{01}$ mode can be converted to $\mathrm{LP}_{11 \mathrm{a}}$ or $\mathrm{LP}_{11 \mathrm{~b}}$ mode with a $3-\mathrm{dB} \mathrm{BW}$ of $2 \mathrm{~nm}$, and the maximum MCEs occurring at the phase-matched wavelength $1557 \mathrm{~nm}$ are $39.6 \mathrm{~dB}$ and $36.4 \mathrm{~dB}$, respectively. The MSPR of the idler parametric CE spectra is larger than $9.2 \mathrm{~dB}$. Thus, there is no harm done to the signal power at the wavelength region of the sidelobes. In addition, the power evolution of the signal and idler along the longitudinal propagation is shown in Fig. 11, which appears to be sinusoidal and agrees well with the theoretical prediction. The signal power in $\mathrm{LP}_{01}$ mode exchanges with the idler power in $\mathrm{LP}_{11 a / b}$ mode, and the complete mode conversion is realized at the end of a 195-m e-HNL-FMF. If the signal propagates over a longer distance, the signal and idler experience small gain, mainly because of the accumulated parametric amplification attributed by the PC, MI, PCc and MIc processes. Furthermore, with a fixed fiber

(a)

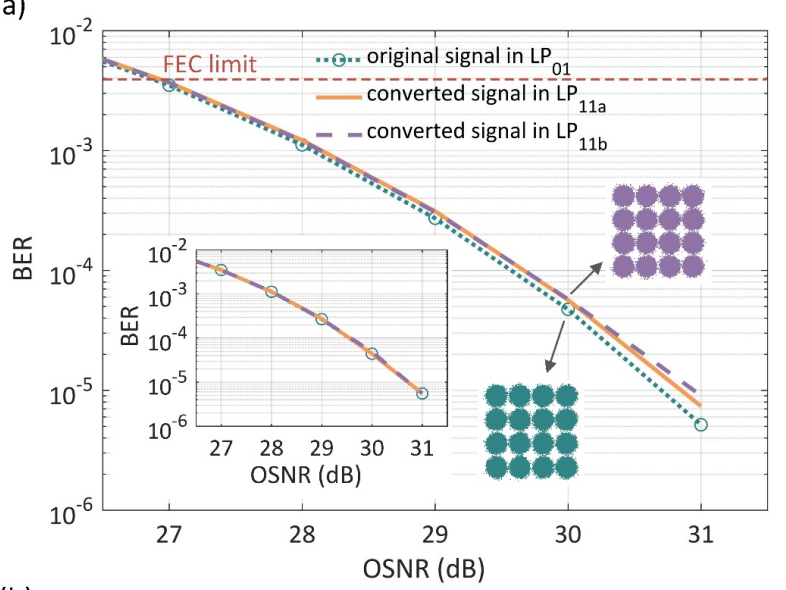

(b)

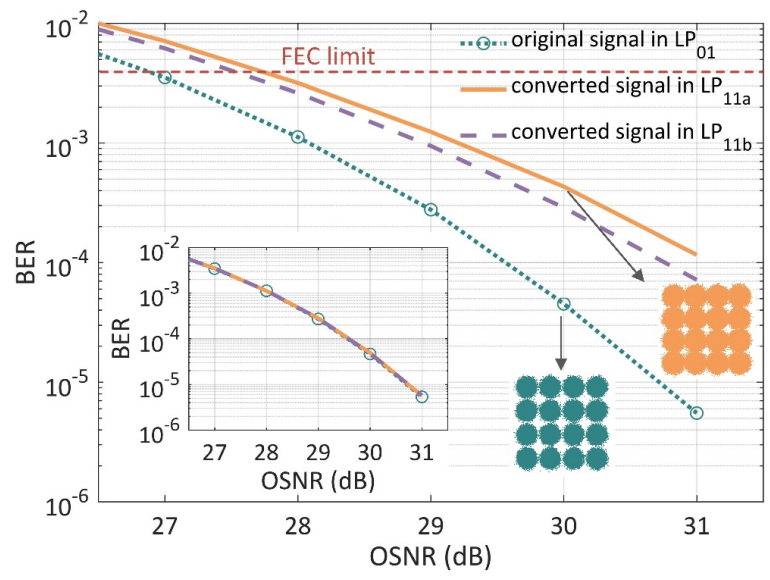

Fig. 12. BER of 100 Gbaud 16-QAM signal before and after mode-selective conversion, when signal wavelength is (a) $1557 \mathrm{~nm}$ and (b) $1565 \mathrm{~nm}$. Inset of each subfigure shows the case when using 25 Gbaud 16-QAM signal. 
length, other applications such as mode multicasting or mode power equalization can be accomplished by the reduction of $P_{p}$ for the specific power ratio of the signal and idler, e.g. a $P_{p}$ of $1.25 \mathrm{~W}$ can be used for mode multicasting where signal and idler powers are equal. In addition, if the MMUX/MDMUX is not ideal, with mode dependent loss, the active mode conversion can be incomplete due to the reduction of $\phi_{N L}$. To overcome such a problem, we can adjust the pump power ratio and enlarge the pump power to ensure the condition that $\phi_{N L}$ equals to $\pi / 2$.

In order to evaluate the performance of active mode-selective conversion, we first introduce a 25 Gbaud 16-QAM signal at $1557 \mathrm{~nm}$ into the $\mathrm{LP}_{01}$ mode. Here, we generate the optical 16-QAM signal modulated by a pseudo random bit sequence (PRBS) with a length of $2^{23}-1$ bits. After the noise loading, the amplitude and phase of each degraded 16-QAM symbol are used as initial states of the signal input. To activate the mode conversion from $L P_{01}$ to $\mathrm{LP}_{11 \mathrm{a}}$ or $L \mathrm{LP}_{11 \mathrm{~b}}$, we use the same setup mentioned above. After the propagation over 195-m e-HNL-FMF, the bit-error rates (BERs) with respect to the optical signal-to-noise ratio (OSNR) are shown in the inset of Fig. 12(a). For the ease of comparison, 100 Gbaud 16-QAM signal is also investigated in Fig. 12(a). The results indicate that, since 25 Gbaud signal has a narrow BW, the active mode-selective conversion bring almost no OSNR penalty. However, for 100 Gbaud 16-QAM signal, the OSNR penalty after the active mode-selective conversion at the hard forward error correction (FEC) threshold of $3.8 \times 10^{-3}$ is less than $0.1 \mathrm{~dB}$. When the signal is at $1565 \mathrm{~nm}$, which means the conversion BW is constrained, accordingly Pump1 and Pump2 should be set at $1532.4 \mathrm{~nm}$ and $1532.1 \mathrm{~nm}$, respectively. The BER performance and constellations of both 100 Gbaud and 25 Gbaud 16-QAM signals are shown in Fig. 12(b) and its inset. We can conclude that, for 25 Gbaud signal, the active mode conversion can be treated as a transparent process. However, as for 100 Gbaud 16-QAM signal, the active mode conversion induced OSNR penalty is $0.9 \mathrm{~dB}$ and $0.7 \mathrm{~dB}$ for the conversion to $\mathrm{LP}_{11 \mathrm{a}}$ and $\mathrm{LP}_{11 \mathrm{~b}}$ modes, respectively. The OSNR penalty can be mitigated by the increase of the pump power and the decrease of the length of the e-HNL-FMF, as shown in Fig. 9.

\section{B. Three-wavelength to three-mode superchannel conversion}

For the three-wavelength to three-mode superchannel conversion, a three-wavelength WDM superchannel signal in $\mathrm{LP}_{01}$ mode is generated, denoted as $s_{0}, s_{1}$ and $s_{2}$, respectively, and centered at the wavelength of $s_{1}$ with an equal frequency spacing. Assuming that $s_{1}$ and $s_{2}$ need to be converted to $\mathrm{LP}_{11 \mathrm{a}}$ mode and $\mathrm{LP}_{11 \mathrm{~b}}$ mode, respectively, while $S_{0}$ remains in $\mathrm{LP}_{01}$ mode with the help of wavelengthsensitivity of WD-BS, two pairs of pumps are required to enable individual active wavelength-selective mode conversions. Pump 1 and Pump 2 denote as $p_{1}$ and $p_{2}$ for the active mode conversion of $s_{1}$ and $s_{2}$, respectively, while their target signal wavelength ranges don’t cover $s_{0}$. However, each
TABLE II

MAIN FWM PROCESSES CONSIDERED IN THREE-WAVELENGTH TO THREE-MODE SUPERCHANNEL CONVERSION

\begin{tabular}{|c|c|}
\hline Type of FWM & FWM process ${ }^{a}$ \\
\hline WD-BS between $p$ and $s$ & $\begin{array}{l}p_{1}{ }^{11 \mathrm{a}}+s_{0 / 1 / 2}{ }^{01} \rightarrow p_{1}{ }^{01}+i_{0 / 1 / 2}{ }^{11 \mathrm{a}} \\
p_{2}{ }^{11 \mathrm{~b}}+s_{0 / 1 / 2}{ }^{01} \rightarrow p_{2}{ }^{01}+i_{0 / 1 / 2}{ }^{11 \mathrm{~b}}\end{array}$ \\
\hline MI between $p$ & $\begin{array}{l}2 p_{1}{ }^{01} \rightarrow p_{2}{ }^{01}+p_{3}{ }^{01} \\
2 p_{2}^{01} \rightarrow p_{1}^{01}+p_{4}{ }^{01}\end{array}$ \\
\hline BS between $p$ & $\begin{array}{l}p_{1}^{11 \mathrm{a}}+p_{2}{ }^{11} \rightarrow p_{1}{ }^{01}+p_{2}{ }^{11 \mathrm{a}} \\
p_{2}{ }^{11 \mathrm{~b}}+p_{1}{ }^{01} \rightarrow p_{2}{ }^{01}+p_{1}{ }^{11 \mathrm{~b}}\end{array}$ \\
\hline PC between $p$ & $\begin{array}{l}p_{1}{ }^{01}+p_{1}{ }^{11 \mathrm{a}} \rightarrow p_{2}{ }^{01}+p_{3}{ }^{11 \mathrm{a}} \\
p_{2}{ }^{01}+p_{2}{ }^{11 \mathrm{~b}} \rightarrow p_{1}{ }^{01}+p_{4}{ }^{11 \mathrm{~b}}\end{array}$ \\
\hline WD-BS between $p$ and $s$, cascaded & $\begin{array}{l}p_{2}{ }^{11 \mathrm{a}}+s_{0 / 1 / 2}{ }^{01} \rightarrow p_{2}{ }^{01}+i_{0 / / 2}{ }^{11 \mathrm{a}} \\
p_{1}{ }^{1 \mathrm{~b}}+s_{0 / 1 / 2}{ }^{01} \rightarrow p_{1}{ }^{01}+i_{0 / 1 / 2}{ }^{11 \mathrm{~b}} \\
p_{1}{ }^{1 \mathrm{~b}}+i_{0 / 1 / 2}{ }^{11 \mathrm{a}} \rightarrow p_{1}{ }^{11 \mathrm{a}}+i_{0 / 1 / 2}{ }^{11 \mathrm{~b}} \\
p_{2}{ }^{1 \mathrm{~b}}+i_{0 / 1 / 2}{ }^{1 \mathrm{a}} \rightarrow p_{2}{ }^{11 \mathrm{a}}+i_{0 / 1 / 2}{ }^{11 \mathrm{~b}} \\
p_{3}{ }^{11 \mathrm{a}}+s_{0 / 1 / 2}{ }^{01} \rightarrow p_{3}{ }^{11}+i_{0 / 1 / 2}{ }^{11 \mathrm{a}} \\
p_{4}{ }^{1 \mathrm{~b}}+s_{0 / 1 / 2}{ }^{01} \rightarrow p_{4}{ }^{01}+i_{0 / 1 / 2}{ }^{11 \mathrm{~b}}\end{array}$ \\
\hline
\end{tabular}

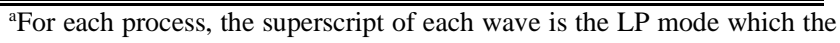
wave is in, and the subscripts of 0/1/2 represent individual processes for 0,1 and 2, respectively.

pump may interact with every signal during the WD-BS process. Thus, we consider all WD-BS processes to investigate the MCE, as well as the mode extinction ratio (MER) which is determined by the power ratio between the target mode and all the other modes. It is worth noting that as the number of waves grows, there are non-negligible FWM processes that occur between the four pump waves, due to their high powers and relatively small frequency spacing. Such processes include MI, BS and PC processes, which will not only cause the pump depletion leading to incomplete mode conversion, but also generate new pump waves introducing the cascaded WD-BS (a)

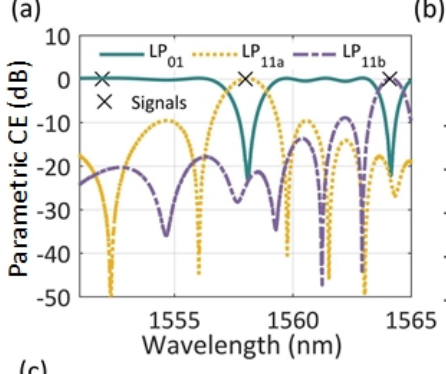

(b)

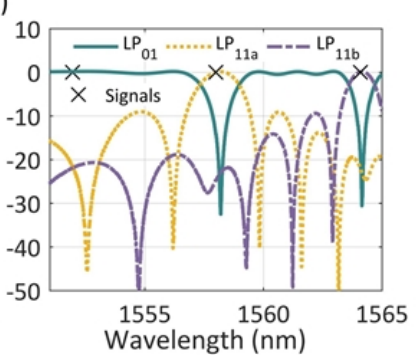

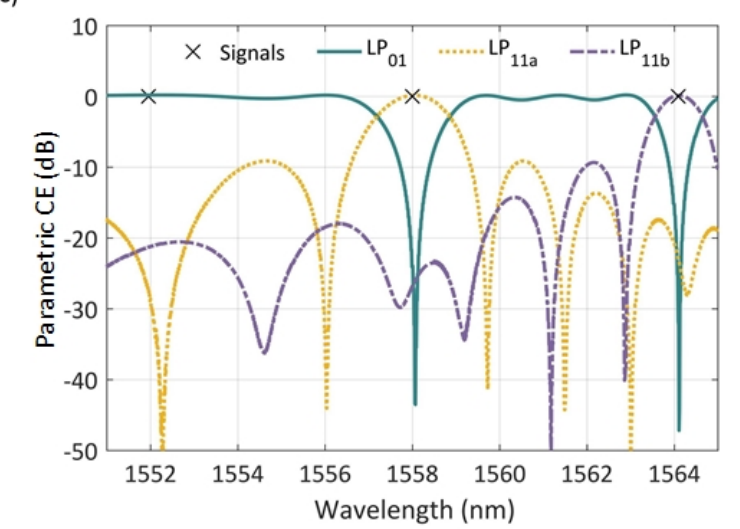

Fig. 13. Parametric CE spectrum of each involved mode for three-wavelength to three-mode superchannel conversion, under condition when (a) considering all FWM processes; (b) ignoring the cascaded WD-BS processes; (c) after the mitigation. 
processes to the signals. The cascaded pumps ( $p_{3}$ and $p_{4}$ ), are generated at $2 \omega_{p_{1}}-\omega_{p_{2}}$ and $2 \omega_{p_{2}}-\omega_{p_{1}}$, respectively. In addition, FWM processes occurring between $\mathrm{LP}_{11 \mathrm{a}}$ and $\mathrm{LP}_{11 \mathrm{~b}}$ modes are also taken into account during the three-mode interaction. All involved waves for the aforementioned FWM processes are summarized in Table II. Note that the intramodal processes among two pumps and a signal are not listed, due to the relatively large wavelength spacing and CD. For given signals centered at $1558 \mathrm{~nm}$ with a frequency spacing of 750 $\mathrm{GHz}$, the wavelengths of $p_{1}$ and $p_{2}$ are $1539.2 \mathrm{~nm}$ and 1533 $\mathrm{nm}$, respectively, and the parametric CE spectra of all three modes are shown in Fig. 13(a). It is worth noting that the wavelength spacing of the three-wavelength superchannel is restricted for independent wavelength operation, because the conversion BW varies with the signal wavelengths, as shown in Fig. 9, which indicates the minimum wavelength spacing for signal at $1558 \mathrm{~nm}$ is $300 \mathrm{GHz}$. From Fig. 13(a) we can observe that the corresponding wavelengths of $s_{1}$ and $s_{2}$, signal power in $\mathrm{LP}_{01}$ mode is depleted while the power is mainly converted to $\mathrm{LP}_{11 \mathrm{a}}$ mode and $\mathrm{LP}_{11 \mathrm{~b}}$ mode, with MCEs of $23.1 \mathrm{~dB}$ and 22.2 $\mathrm{dB}$, and MERs of $20.4 \mathrm{~dB}$ and $19.7 \mathrm{~dB}$, respectively. At the same time, almost no power penalty is introduced to $s_{1}$ with a MER of $21.1 \mathrm{~dB}$. However, such performance results are degraded by the FWM processes between pumps and the cascaded WD-BS processes. Among those processes, the first four cascaded WD-BS processes in Table II caused by the BS processes between pumps contribute to the most of performance degradation, because in case a WD-BS process is phase-matched between $\mathrm{LP}_{01}$ and one of $\mathrm{LP}_{11}$ modes, the cascaded WD-BS at the same wavelengths between $\mathrm{LP}_{01}$ and the other $\mathrm{LP}_{11}$ mode has a high chance to be phase-matched, due to the similar $\beta_{1}$ properties of $\mathrm{LP}_{11}$ modes. The same condition happens for the WD-BS between the $\mathrm{LP}_{11}$ modes. In comparison, if we ignore such cascaded WD-BS processes, the parametric CE spectra are shown in Fig. 13(b). The BS-generated pumps leads to an MCE degradation of around 9 $\mathrm{dB}$ for both $s_{1}$ and $s_{2}$, which further causes MER deterioration of $2 \mathrm{~dB}$ and $2.6 \mathrm{~dB}$ for $s_{1}$ and $s_{2}$, respectively. Such an effect can be compensated by adjusting the power of Pump1 and Pump2, as shown in Fig. 13(c). When Pump1 power into $\mathrm{LP}_{11 \text { a }}$ mode is increased by $0.37 \mathrm{~dB}$ while Pump2 power into $\mathrm{LP}_{01}$ mode is attenuated by $0.36 \mathrm{~dB}$, the conversion has the best performance, with MCEs of $43.5 \mathrm{~dB}$ and $47.2 \mathrm{~dB}$ for $s_{1}$ and $s_{2}$, and MERs of $21 \mathrm{~dB}, 26 \mathrm{~dB}$ and $24.1 \mathrm{~dB}$ for $s_{0}$, $s_{1}$ and $s_{2}$, respectively. If we consider mode crosstalk of the MMUX, adjusting either the pump power or power ratio with similar procedure is helpful to mitigate such impairment. Although the MMUX induced mode crosstalk is almost independent of the WD-BS process, the MMUX with lower mode crosstalk is ideal to improve the overall performance.

Next, under the conditions of the optimal pump powers, $3 \times 25$ Gbaud 16-QAM signals are introduced into the e-HNL-FMF based OXC to perform the three-wavelength to

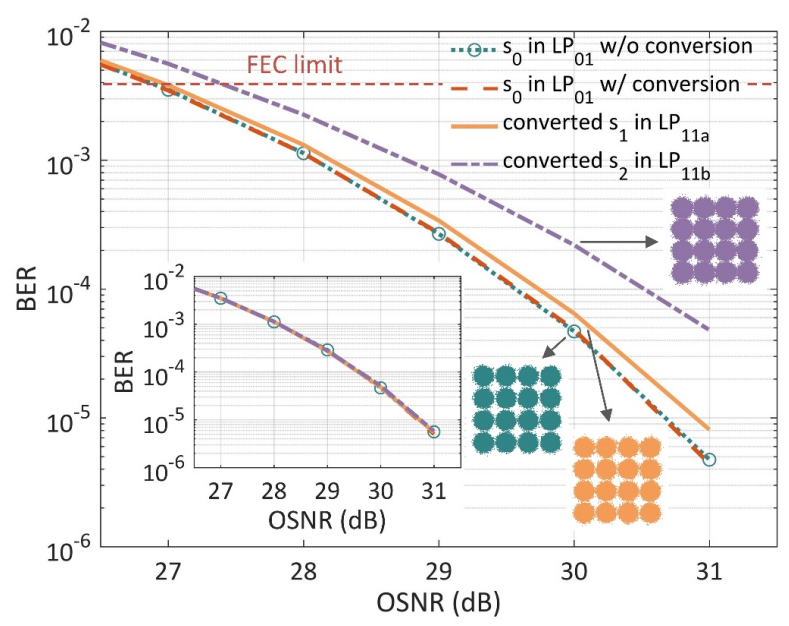

Fig. 14. BERs and constellations of $3 \times 100$ Gbaud $16-Q A M$ signals before and after three-wavelength to three-mode superchannel conversion. Inset shows the case when $3 \times 25$ Gbaud 16-QAM signals are used.

three-mode superchannel conversion. The wavelength and mode of each pump and signal is the same as the optimal configuration of the CW case. After propagation over 195-m e-HNL-FMF, the BER performance of each signal before and after the three-wavelength to three-mode superchannel conversion is shown in the inset of Fig. 14. BERs of all three signals remain almost the same, indicating the error-free operation of the three-wavelength to three-mode superchannel conversion. For the case of $3 \times 100$ Gbaud $16-Q A M$ signals with the same wavelength and power configuration, the BER performance is shown in Fig. 14. The BER performance of $s_{0}$ still remains the same, before and after the three-wavelength to three-mode superchannel conversion due to the flat passband property, while the OSNR penalties at the hard FEC threshold for $s_{1}$ and $s_{2}$ are $0.1 \mathrm{~dB}$ and $0.5 \mathrm{~dB}$, respectively. The performance degradation of $s_{2}$ is more severe than that of $s_{1}$, due to the long wavelength induced narrow conversion BW.

\section{CONCLUSIONS}

We propose an active mode-selective conversion scheme based on the intermodal WD-BS process, without the parasitic wavelength conversion that exists in most intermodal FWM processes. The phase-matching condition and complete mode conversion condition are numerically identified. Meanwhile, we design a novel e-HNL-FMF whose modal dispersions are engineered to satisfy the phase-matching condition of active mode-selective conversion, while obtaining relatively high intermodal nonlinear coefficients of $3.23(\mathrm{~W} \cdot \mathrm{km})^{-1}$ between $\mathrm{LP}_{01}$ and $\mathrm{LP}_{11 \mathrm{a}}$ modes and $3.14(\mathrm{~W} \cdot \mathrm{km})^{-1}$ between $\mathrm{LP}_{01}$ and $\mathrm{LP}_{11 \mathrm{~b}}$ modes. After performance optimization, a 195-m e-HNL-FMF is used for the OXC targeted at WDM-MDM networks. Through the management of the wavelengths and the launched modes of the pumps, both active mode-selective conversion and three-wavelength WDM superchannel to three-mode MDM superchannel conversion can be realized with a 3-dB BW of more than $100 \mathrm{GHz}$ over the C-band. The proposed e-HNL-FMF can realize other potential OXC 
applications in terms of mode multicasting, mode filtering, as well as wavelength exchange, for all-optical signal processing in WDM-MDM networks.

\section{REFERENCES}

[1] T. Torounidis, P. A. Andrekson and B. E. Olsson, "Fiber-optical parametric amplifier with 70-dB gain," IEEE Photon. Technol. Lett., vol. 18, no. 10, pp. 1194-1196, May 2006.

[2] S. Oda, H. Sunnerud, and P. A. Andrekson, "High efficiency and high output power fiber-optic parametric amplifier,” Opt. Lett., vol. 32, no. 13, pp. 1776-1778, 2007.

[3] G. W. Lu, T. Sakamoto and T. Kawanishi, "Coherently-pumped FWM in HNLF for 16QAM wavelength conversion free of phase noise from pumps,” in Proc. Eur. Conf. Opt. Commun., 2014, paper P.1.16.

[4] M. E. Marhic, Y. Park, F. S. Yang, and L. G. Kazovsky, "Widely tunable spectrum translation and wavelength exchange by four-wave mixing in optical fibers,” Opt. Lett., vol. 21, no. 23, pp. 1906-1908, 1996.

[5] K. K. Y. Wong, M. E. Marhic, K. Uesaka and L. G. Kazovsky, "Demonstration of wavelength exchange in a highly-nonlinear fiber," in Proc. Eur. Conf. Opt. Commun., 2001, paper We.L.1.3.

[6] K. Uesaka, K. K. Y. Wong, M. E. Marhic, and L. G. Kazovsky, "Wavelength exchange in a highly nonlinear dispersion-shifted fiber: theory and experiments,” IEEE J. Sel. Top. Quantum Electron., vol. 8, no. 3, pp. 560-568, 2002.

[7] R. Slavik, F. Parmigiani, J. Kakande, C. Lundström, M. Sjodin, P. A. Andrekson, R. Weerasuriya, S. Sygletos, A. D. Ellis, L. Grüner-Nielsen, D. Jakobsen, S. Herstrom, R. Phelan, J. O'Gorman, A. Bogris, D. Syvridis, S. Dasgupta, P. Petropoulos, and D. J. Richardson, "All-optical phase and amplitude regenerator for next-generation telecommunications systems," Nat. Photonics, vol. 4, no. 10, pp. 690-695, 2010.

[8] Y. Meng, J. Lian, S. Fu, M. Tang, P. Shum and D. Liu, "All-Optical DPSK Regenerative One-to-Nine Wavelength Multicasting Using Dual-Pump Degenerate Phase Sensitive Amplifier,” J. Lightw. Technol., vol. 32, no. 15, pp. 2605-2612, 2014.

[9] P. A. Andrekson, H. Sunnerud, S. Oda, T. Nishitani, and J. Yang, "Ultrafast, atto-Joule switch using fiber-optic parametric amplifier operated in saturation,” Opt. Express, vol. 16, no. 15, pp. 10956-10961, 2008.

[10] R. Nissim, A. Pejkic, E. Myslivets, B. P. Kuo, N. Alic, and S. Radic, "Ultrafast optical control by few photons in engineered fiber," Science, vol. 345, no. 6195, pp. 417-419, 2014.

[11] J. Gao, Y. Meng, S. Fu, M. Karlsson, P. A. Andrekson, M. Tang, and D. Liu, "Theoretical Investigation of Longitudinal Dispersion Fluctuations on all-fiber phase-sensitive parametric optical switch,” J. Lightw. Technol., vol. 35, no. 9, pp. 1646-1653, 2017.

[12] D. J. Richardson, J. M. Fini, and L. E. Nelson, “Space-division multiplexing in optical fibres,” Nat. Photonics, vol. 7, no.5, pp. 354-362, 2013.

[13] R. Ryf, S. Randel, A. H. Gnauck, C. Bolle, A. Sierra, S. Mumtaz, M. Esmaeelpour, E. C. Burrows, R. J. Essiambre, P. J. Winzer, D. W. Peckham, A. H. McCurdy, and R. Lingle, "Mode-division multiplexing over $96 \mathrm{~km}$ of few-mode fiber using coherent $6 \times 6$ MIMO processing," $J$. Lightw. Technol., vol. 30, no. 4, pp. 521-531, 2012.

[14] N. Zhao, B. Huang, R. Amezcua-Correa, X. Li, and G. Li, "Few-mode fiber optical parametric amplifier,” in Proc. Opt. Fiber Commun. Conf. (Opt. Soc. Amer.), 2013, paper OTu2D.5.

[15] E. Nazemosadat, A. Lorences-Riesgo, M. Karlsson, and P. A. Andrekson, "Design of highly nonlinear few-mode fiber for C-Band optical parametric amplification,” J. Light. Technol., vol. 35, no. 14, pp. 2810-2817, 2017.

[16] Y. Xiao, R. J. Essiambre, M. Desgroseilliers, A. M. Tulino, R. Ryf, S. Mumtaz, and G. P. Agrawal, "Theory of intermodal four-wave mixing with random linear mode coupling in few-mode fibers," Opt. Express, vol. 22, no. 26, pp. 32039-32059, 2014.

[17] R. J. Essiambre, M. A. Mestre, R. Ryf, A. H. Gnauck, R. W. Tkach, A. R. Chraplyvy, Y. Sun, X. Jiang, and R. Lingle, "Experimental investigation of inter-modal four-wave mixing in few-mode fibers," IEEE Photonics Technol. Lett., vol. 25, no. 6, pp. 539-542, 2013.

[18] F. Parmigiani, Y. Jung, P. Horak, L. Grüner-Nielsen, T. Geisler, P. Petropoulos, and D. Richardson, "C- to L-band wavelength conversion enabled by parametric processes in a few mode fiber," in Proc. Opt. Fiber Commun. Conf. (Opt. Soc. Amer.), 2017, paper Th1F.4.

[19] O. F. Anjum, P. Horak, Y. Jung, M. Suzuki, Y. Yamamoto, T. Hasegawa, P. Petropoulos, D. J. Richardson, and F. Parmigiani, "Bandwidth enhancement of inter-modal four wave mixing Bragg scattering by means of dispersion engineering," APL Photonics, vol. 4, no. 2, 2019.

[20] J. Gao, E. Nazemosadat, J. Schröder, C. Yang, S. Fu, M. Tang, D. Liu, W. Tong, M. Karlsson, and P. A. Andrekson, "Vector Mode Inter-Modal Wavelength Conversion in a Dispersion Tailored Highly Nonlinear Few-Mode Fibre,” in Proc. Eur. Conf. Opt. Commun., 2019, paper P38.

[21] G. Rademacher, R. S. Luís, B. J. Puttnam, Y. Awaji, M. Suzuki, T. Hasegawa, and N. Wada, "Wide-Band Intermodal Wavelength Conversion in a Dispersion Engineered Highly Nonlinear FMF," in Proc. Opt. Fiber Commun. Conf. (Opt. Soc. Amer.), 2019, paper W1C.4.

[22] H. Zhang, M. Bigot-Astruc, L. Bigot, P. Sillard, and J. Fatome, "Multiple modal and wavelength conversion process of a 10-Gbit/s signal in a 6-LP-mode fiber,” Opt. Express, vol. 27, no. 11, pp. 15413-15425, 2019.

[23] J. Gao, E. Nazemosadat, C. Yang, S. Fu, M. Tang, W. Tong J. Carpenter, J. Schroder, M. Karlsson, and P. A. Andrekson, "Design, fabrication, and characterization of a highly nonlinear few-mode fiber,” Photonics Res., vol. 7, no. 11, pp. 1354-1362, 2019.

[24] E. Nazemosadat and A. Mafi, "Design considerations for multicore optical fibers in nonlinear switching and mode-locking applications,” J. Opt. Soc. Am. B, vol. 31, no. 8, pp. 1874-1878, 2014.

[25] K. Inoue, "Four-Wave Mixing in an Optical Fiber in the Zero-Dispersion Wavelength Region,” J. Light. Technol., vol. 10, no. 11, pp. 1553-1561, 1992.

[26] G. P. Agrawal, Nonlinear Fiber Optics, 5th ed. San Diego, CA: Academic, 2013.

[27] S. M. M. Friis, I. Begleris, Y. Jung, K. Rottwitt, P. Petropoulos, D. J. Richardson, P. Horak, and F. Parmigiani, "Inter-modal four-wave mixing study in a two-mode fiber," Opt. Express, vol. 24, no.26, pp. 30338-30349, 2016.

[28] C. J. McKinstrie, S. Radic, and A. R. Chraplyvy, "Parametric Amplifiers Driven by Two Pump Waves,” IEEE J. Sel. Top. Quantum Electron., vol. 8, no. 3, pp. 538-547, 2002.

[29] Z. Wu, J. Li, D. Ge, F. Ren, P. Zhu, Q. Mo, Z. Li, Z. Chen, and Y. He, "Demonstration of all-optical MDM/WDM switching for short-reach networks," Opt. Express, vol. 24, no. 19, pp 21609-21618, 2016.

[30] K. Nakajima and M. Ohashi, "Dopant dependence of effective nonlinear refractive index in GeO2- and F-doped core single-mode fibers," IEEE Photonics Technol. Lett., vol. 14, no. 4, pp. 492-494, 2002. 